Al-Azhar Bull. Sci. Vol. 24, No. 2 (Dec.): pp. 1-15, 2013.

\title{
PHYSICO-CHEMICAL CHARACTERISTICS AND NATURAL RADIOACTIVITY OF ISMAILIA CANAL WATER, RIVER NILE, EGYPT.
}

ABDEL RAZEQ S.A. ${ }^{1}$, SOLIMAN S.A. ${ }^{2}$, BAKR W. ${ }^{3}$, ABDEL GHAFAR I ${ }^{4}$.

1,4. Analytical Chemistry Department, Faculty of Pharmacy, Al-Azhar University, Cairo, Egypt.

2. Chemistry Department, Faculty of Science, Al-Azhar University, Cairo, Egypt.

3. Radiation Chemistry Department, National Center for Nuclear Safety and Radiation Control, Cairo, Egypt.

\begin{abstract}
Ismailia Canal is considered as one of the most important drinking and irrigation water source in Egypt. Twenty four water samples were collected during four successive seasons from summer 2010 to spring 2011 along the area extending from El Mazallat to Anshas . Physical parameters temperature, E.C and TDS and chemical parameters $\mathrm{pH}$,alkalinity, TH, DO, COD, BOD, $\mathrm{NH}_{3}, \mathrm{NO}_{2}^{-}, \mathrm{NO}_{3}^{-}, \mathrm{PO}_{4}^{-3}, \mathrm{Cl}^{-}, \mathrm{F}^{-}, \mathrm{Br}^{-}, \mathrm{SO}_{4}{ }^{-2}, \mathrm{Ca}^{2+}, \mathrm{Mg}^{2+}, \mathrm{Na}^{+}, \mathrm{K}^{+}, \mathrm{Fe}^{+3}$, $\mathrm{Cd}^{+2}, \mathrm{Zn}^{+2}, \mathrm{Cu}^{+2}, \mathrm{~Pb}^{+2}$, and $\mathrm{Al}^{+3}$ were analyzed to identify water quality of Ismailia Canal. The results of these analyses, when compared with Egyptian and international standards were found to be within the permissible limits except $\mathrm{COD}, \mathrm{NH}_{3}, \mathrm{Fe}^{+3}, \mathrm{Cd}^{+2}, \mathrm{~Pb}^{+2}$, and $\mathrm{Al}^{+3}$ at certain sites and/ or certain seasons. Additionally, the naturally occurring radioactive materials ${ }^{238} \mathrm{U},{ }^{232} \mathrm{Th}$ and $\left.{ }^{40} \mathrm{~K}\right)$ were analyzed where ${ }^{238} \mathrm{U}$ and ${ }^{232} \mathrm{Th}$ were found to exceed EPA limit. Finally water quality index (WQI) was calculated to be 80.6 and 84.3 according to Egyptian higher committee of water and WHO guidelines, respectively; this indicates that the water quality of Ismailia Canal was good.
\end{abstract}

\section{Introduction}

Rivers play a major role in assimilating or carrying industrial, municipal waste water and agricultural run-off which are major sources of pollution that affecting the water quality (Nair et al) ${ }^{1}$.

Ismailia Canal is considered as one of the largest fresh water canals branched from the River Nile. It receives a lot of industrial waste water which leading to undesirable effects on its water quality (Geriesh et $a l^{2}$., Abdo et al ${ }^{3}$., and Abd ElHady and Hussian ${ }^{4}$ ).

Stahl and Ramadan, ${ }^{5}$ studied inorganic chemical water quality of Ismailia Canal. $\mathrm{Fe}, \mathrm{Mn}$ and $\mathrm{Zn}$ were found in low concentrations. The amounts of dissolved salts were still below the "German Trinkwasserverordnung." A higher total organic carbon level indicated the presence of organic contaminants. While the natural radioactivity level was in the normal range.

Abdo et al., ${ }^{3}$ determined physical and chemical characteristics of Ismailia Canal water and concluded that the main pollution sources of the canal were the domestic and effluents of police camp and petroleum companies. 
The quality of water is now the concern of experts in all countries. The water quality of any source depends on its location and environmental protection. Thus, this study was devoted to assess the quality and nature of Ismailia Canal water through physical and chemical analyses together with natural radioactivity.

\section{Experimental}

\subsection{Sampling}

Water samples were collected seasonally from Ismailia Canal during the period from summer 2010 to spring 2011. Six sites were selected along the canal extending from El Mazallat square to Anshas, Table(1) and Figure (1).

Water samples were collected at $60 \mathrm{~cm}$ depth each of $2 \mathrm{~L}$ in polyethylene containers in an ice-box. Samples for heavy metals analysis were preserved with concentrated nitric acid. Samples for determination of dissolved oxygen (DO) and biochemical oxygen demand (BOD) were collected in glass stoppered oxygen bottles. For natural radioactivity measurement, one liter filtered water samples were transferred into one liter Marinillie beaker, carefully sealed and stored for 4 weeks ( Mollahet al., ${ }^{6}$ ).

All selected parameters were measured according to $\mathrm{APHA}^{7}$, (2005) except where noted. Both electrical conductivity (E.C) and total dissolved solids (TDS) were determined by conductivity meter (Con 510, USA). pH values were measured using $\mathrm{pH}$ meter (Cyber scan 2100, Singapore). Total alkalinity was estimated by titration with standard $0.02 \mathrm{~N} \mathrm{H}_{2} \mathrm{SO}_{4}$ using phenolphthalein and bromo cresol green as indicators. Total hardness (TH) was determined by EDTA titration method. DO measurement was done by Winkler method and ion selective electrode (Jenway 9500, UK), COD by dichromate oxidation method and BOD by 5-day incubation method.Concentration levels of $\mathrm{NH}_{3}, \mathrm{NO}_{2}{ }^{-}, \mathrm{NO}_{3}{ }^{-}$and $\mathrm{PO}_{4}{ }^{3-}$ were determined using colorimetric methods: phenate, azo-dye, reduction into nitrite followed by azo-dye formation and stannous chloride methods, respectively using Shimadzu, UV-Vis 1601PC spectrophotometer,Japan. Anions $\left(\mathrm{F}^{-}, \mathrm{Cl}^{-}, \mathrm{Br}^{-}\right.$and $\left.\mathrm{SO}_{4}^{-2}\right)$ and cations $\left(\mathrm{Ca}^{2+}\right.$ , $\mathrm{Mg}^{2+}, \mathrm{Na}^{+}$and $\mathrm{K}^{+}$) were measured by ion chromatography (Dionex ICS$3000, \mathrm{USA})$. $\mathrm{Al}$ and heavy metals $(\mathrm{Pb}, \mathrm{Cu}, \mathrm{Cd}, \mathrm{Zn})$ by $\mathrm{AAS}$. Iron was measured by IC (Dionex, ${ }^{8}$ ) and through colorimetric method .Natural radioactivity determination was carried out by $\gamma$ - ray spectrometer with hyper pure germanium detector, Canbberra type . 


\section{Results and Discussion:}

Ismailia Canal has its inlet from the Nile at north of Cairo at El-Mazalet region and runs down town of Ismailia where it bifurcates to Port Said and Suez.

It transport about five million cubic meters per day for drinking, industrial and irrigation purposes (Abdo et al., ${ }^{3}$ and Abdo and El-Nasharty ${ }^{9}$ ).

In this study six sites were selected for water analysis along the first $27 \mathrm{Km}$ of the canal where the largest industrial zone was located, Table (1) and Figure (1).

The physical-chemical parameters are considered as the most important principles in the detection of the quality of water. Variations of the physic-chemical characteristics of Ismailia Canal water during summer, autumn, winter and spring were presented in Tables (2-6) and these results were compared with $\mathrm{EHCW}^{10}$, Egyptian $\mathrm{Law}^{11}(48 / 1982), \mathrm{WHO}^{12}, \mathrm{EPA}^{13}$ and law of Council of the European Union $^{14}($ EU), Table (7)

\subsection{Physical parameters}

Water temperature $\left(15.0-28^{\circ} \mathrm{C}\right)$ showed clear seasonal trends with slight variations between different sites due to different sampling times, climatic conditions and humidity.

TDS and E.C ranges were $240-135 \mathrm{mg} / \mathrm{L}$ and $480-270 \mu \mathrm{S} / \mathrm{cm}$, respectively. Decay and degradation of most microorganism species in the lower water level during drought period (Abdo ${ }^{15}$, and Abdo and El-Nasharty ${ }^{9}$ ) leaded to obvious increase in TDS values in winter which was reflected in results of E.C .

\subsection{Chemical parameters}

\subsection{1. pH ,Alkalinity and Total Hardness}

$\mathrm{pH}$ of water of Ismailia Canal was slightly alkaline (7.0-8.2) with slight increase in spring nearly in all investigated sites which may be ascribed to the dense of vegetation and phytoplankton, followed by increased photosynthetic activity and consumption of $\mathrm{CO}_{2}\left(\right.$ Abdel-Satar ${ }^{16}$ and $\mathrm{Sabae}^{17}$ ).

Alkalinity ranged between 170 and $190 \mathrm{mg} / \mathrm{L} \mathrm{CaCO}_{3}$ and Ismailia Canal water was found to be bicarbonate water stream and this agreed with the results reported by Abdel- Malik et $\mathrm{al}^{18}$.

Hardness of Ismailia Canal water ranged between 120 and $152 \mathrm{mg} / \mathrm{L}$ $\mathrm{CaCO}_{3}$; thus it can be considered as slightly hard according to EPA ${ }^{13}$. 


\subsubsection{DO, COD and BOD}

DO showed maximum average value in winter $(9.53 \mathrm{mg} / \mathrm{L})$ and minimum in summer $(5.9 \mathrm{mg} / \mathrm{L})$; the latter low value may be due to the lower capacity of water to hold oxygen at higher temperature (Murugesan and Rajakumari ${ }^{19}$ and Jayakumar et al. ${ }^{20}$ ). Ismailia Canal water exhibited COD values in the range of $10.4-30 \mathrm{mg} / \mathrm{L}$ with high fluctuations at six sites which mostly exceeded the limits of Egyptian Law $^{11}$ (Less than $15 \mathrm{mg} / \mathrm{L}$ ) which indicated heavy load of pollution along the canal. The observed values of BOD ranged from 0.0 to $3.7 \mathrm{mg} / \mathrm{L}$ with highest average value in winter as a result of the presence of high amount of organic matter especially during drought period, resulting in the uptake of oxygen in the oxidative breakdown of them (Abdel-Razak et al) ${ }^{21}$.

\subsubsection{Nutrients}

Nitrogen (in the form of $\mathrm{NH}_{3}, \mathrm{NO}_{2}{ }^{-}$and $\mathrm{NO}_{3}{ }^{-}$) and phosphorus are the nutrients most commonly identified as pollutants (Igbinosa and Okoh) ${ }^{23} \cdot \mathrm{NH}_{3}(0.148 \mathrm{mg} / \mathrm{L}$ ) contents ranged from 0.0 to $0.664 \mathrm{mg} / \mathrm{L}$ with maximum average value in summer which may be due to the fact that the high temperature accelerates the reduction rate of nitrate into ammonia (Abdo et al) ${ }^{3}$. Again the maximum values of $\mathrm{NH}_{3}$ at site II might be a result of effluent from iron and steel mills $(\mathrm{CCME})^{24}$.

The range of $\mathrm{NO}_{2}^{-}$in Ismailia Canal water was $0-58.1 \mu \mathrm{g} / \mathrm{L}$ with relatively high average value in autumn $(44.11 \mu \mathrm{g} / \mathrm{L})$ and winter $(46.08 \mu \mathrm{g} / \mathrm{L})$ which are mainly attributed to the oxidation of existing ammonia, yielding $\mathrm{NO}_{2}^{-}$, as intermediate state especially in abundant oxygen during winter (Wetzel ${ }^{25}$ and $\left.\mathrm{Abdo}^{26}\right)$. There is also an increase of $\mathrm{NO}_{2}{ }^{-}$concentration at site II which may be ascribed to oxidation of part of ammonia released as effluent from iron and steel mills.

The concentration of $\mathrm{NO}_{3}^{-}$varied from 0 to $2.5 \mathrm{mg} / \mathrm{L}$ with decreased concentration in spring for most sites which might be ascribed to the uptake of $\mathrm{NO}_{3}^{-}$by natural phytoplankton and its reduction by denitrifying bacteria (Sabae andAbdel-Satar ${ }^{27}$ and Abdel-Satar ${ }^{16}$ ).

$\mathrm{PO}_{4}{ }^{3-}$ is present in natural water as soluble phosphates and organic phosphates. Soluble $\mathrm{PO}_{4}{ }^{3-}$ in Ismailia Canal water was found to be in the range of 0 to 0.821 $\mathrm{mg} / \mathrm{L}$. As illustrated in Table (2-6), high concentration of $\mathrm{PO}_{4}{ }^{3-}$ was clear at site $\mathrm{V}$ in the four seasons which may be due to presence of the discharge point of the Abu Za'baal phosphate fertilizer factory at this site. 


\subsubsection{Anions}

$\mathrm{Cl}^{-}, \mathrm{F}^{-}, \mathrm{Br}^{-}$and $\mathrm{SO}_{4}{ }^{-2}$ levels in Ismailia Canal water were 12.2 - $40.4,0.81$ 1.06, $0-0.60$ and $16.6-39.7 \mathrm{mg} / \mathrm{L}$, respectively. $\mathrm{Cl}^{-}$reached a maximum concentration of about $41.0 \mathrm{mg} / \mathrm{L}$ in winter as a result of low levels of water during draught period. $\mathrm{F}^{-}$showed high values at sites $\mathrm{V}$ and VI due to presence of phosphate fertilizer factory where fluorapatite (fluorinated calcium phosphate rock) was used as the primary source of phosphate fertilizer (Haamer) ${ }^{28}$. However, all values were within international limits but exceeded the limits of $\mathrm{EHCW}^{10} . \mathrm{Br}^{-}$ showed slight fluctuations between different seasons and sites. Whereas the relative increase in $\mathrm{SO}_{4}^{-2}$ concentrations during winter may be due to death and decomposition of aquatic microorganisms with aconsequent oxidation of the liberated sulfur into sulfate in presence of high DO in this season (Abdo et al) ${ }^{3}$.

\subsubsection{Cations}

All cations $\left(\mathrm{Ca}^{2+}, \mathrm{Mg}^{2+}, \mathrm{Na}^{+}\right.$and $\left.\mathrm{K}^{+}\right)$showed small variations in their levels at the six sites of Ismailia Canal over 4 seasons. They varied in the range of 23.8 31.6, 16.2 - 10.6, 23.1 - 34.4 and 3.89 - $5.9 \mathrm{mg} / \mathrm{L}$, respectively.

\subsubsection{Heavy metals and aluminium}

Ismailia Canal water samples were analyzed for heavy metals $(\mathrm{Fe}, \mathrm{Cd}, \mathrm{Cu}, \mathrm{Zn}$ and $\mathrm{Pb}$ ) and $\mathrm{Al}$. Iron contents ranged between 0 and $0.41 \mathrm{mg} / \mathrm{L}$. Some $\mathrm{Fe}$ concentrations were exceeded $\operatorname{EPA}^{13}(0.2 \mathrm{mg} / \mathrm{L})$ and $\operatorname{EU}^{14}(0.3 \mathrm{mg} / \mathrm{L})$ especially at site VI (Anshas, near the fertilizer factory) due to presence of iron as impurities in phosphate rock used in manufacture of phosphate fertilizer (Arlow) ${ }^{29}$.

$\mathrm{Cd}$ range was $1.0-12.4 \mu \mathrm{g} / \mathrm{L}$ with lowest values in spring which might be attributed to sorption of metals on suspended particles where removal as fine colloidal occur at high $\mathrm{pH}$ in this season (Toufeek) ${ }^{30}$. Some values of Cd were found to be higher than guideline values approved by $\mathrm{EHCW}{ }^{10}, \mathrm{EPA}^{13}, \mathrm{EU}^{14}$ $(5 \mu \mathrm{g} / \mathrm{L})$ and WHO ${ }^{12}$ ( $\left.3 \mu \mathrm{g} / \mathrm{L}\right)$ which may be related to the presence of steel factory at site II of the canal.

Undetectable $\mathrm{Cu}, \mathrm{Zn}$ and $\mathrm{Pb}$ levels were mostly at all investigated sites in the four seasons except for $\mathrm{Pb}$ which exceeded guidelines of $\mathrm{EHCW}{ }^{10}(50 \mu \mathrm{g} / \mathrm{L})$, $\mathrm{WHO}^{12}(10 \mu \mathrm{g} / \mathrm{L}), \mathrm{EPA}^{13}(10 \mu \mathrm{g} / \mathrm{L})$ and $\mathrm{EU}^{14}(15 \mu \mathrm{g} / \mathrm{L})$ during winter due to the drop of water levels .

$\mathrm{Al}$ values varied in range of $0-1.383 \mathrm{mg} / \mathrm{L}$. Its values were mostly higher than permissible values of $(0.2 \mathrm{mg} / \mathrm{L})$ of $\mathrm{EHCW}^{10}, \mathrm{WHO}^{12}, \mathrm{EPA}^{13}$ and $\mathrm{EU}^{14}$. This may 
be ascribed to domestic wastes along canal. It is worthy to note that the highest values were especially at sites $\mathrm{V}$ and $\mathrm{VI}$ as $\mathrm{Al}$ is one of the impurities in phosphate rock used in fertilizer manufacture (Arlow, ${ }^{29}$.

\subsection{Naturally occurring radioactive materials}

${ }^{238} \mathrm{U},{ }^{232} \mathrm{Th}$ and ${ }^{40} \mathrm{~K}$ are three long-lived naturally occurring radionuclides present in the earth crust. The activity concentration levels were found to range between lower than detection limit (DL) and 103.84, 6.97 and $3.65 \beta \mathrm{q} / \mathrm{L}$ for the three nucleoids, respectively. The lowest values were found in samples collected from the entrance of the canal for the three determined isotops. ${ }^{238} \mathrm{U}$ showed increase in samples collected from the front of petroleum company where oil and gas extraction and processing operation sometimes accumulate naturally occurring radioactive materials (Smith, $)^{31}$.There was also increase of ${ }^{232} \mathrm{Th}$ in samples collected from the front of Nuclear Research Centre which was very near to Abu Za'bal fertilizer factory and $40 \mathrm{~K}$ in samples from the front of the same factory due to that the effluents of this factory were probably the main origin of natural radioactivity in this area (Stahl and Ramadan $)^{5}$.

${ }^{238} \mathrm{U}$ was found to exceed the guideline value approved by $\operatorname{EPA}^{13}(0.372 \beta \mathrm{q} / \mathrm{L})$. ${ }^{232} \mathrm{Th}$ was also exceeded the guideline of EPA ${ }^{13}(1 \beta \mathrm{q} / \mathrm{L})$ which may be a result of human activities caused by agriculture drainage or industrial effluent.

\subsection{Water Quality Index}

Water Quality Index (WQI) is an arithmetic tool used to transform large quantities of water quality data into a single cumulatively derived number (Kalraet $a l .,)^{32}$ It is used to assess water quality relative to the standard for domestic use and to provide insight into the degree to which water quality is affected by human activity (Abd-El-Razak et al., $)^{21}$.

The Canadian WQI (CCME) $)^{33}$ uses simple calculation and hence it was used in this study to evalute water of Ismailia Canal. WQI of Ismailia Canal water was calculated to be 80.6 and 84.3 (according to Egyptian and WHO guidelines) indicating that water quality could be considered as good.

\section{Conclusion}

The increased values of some chemical parameters of water of Ismailia Canal at certain sites especially $\mathrm{COD}, \mathrm{NH}_{3}, \mathrm{Fe}, \mathrm{Cd}, \mathrm{Pb}$, and $\mathrm{Al}$ represented sources of pollution of the canal. This pollution was due to effluents of petroleum, steel and fertilizer factories as well as domestic wastes .Therefore, treatment of waste water effluents become a must before drainage into the canal. 
PHYSICO-CHEMICAL CHARACTERISTICS AND NATURAL .......

Table (1): Sampling Sites of Ismailia Canal.

\begin{tabular}{|c|c|c|c|}
\hline Site No & Site location & $\begin{array}{l}\text { Distance from entrance of the } \\
\text { canal }\end{array}$ & GPS data \\
\hline I & $\begin{array}{l}\text { Entrance of Ismailia } \\
\text { Canal }\end{array}$ & $0 \mathrm{Km}$ & $\begin{array}{l}\text { N: } 30^{\circ} 6^{\prime} 5,12^{\prime \prime} \\
E: 31^{\circ} 14^{\prime} 7,34^{\prime \prime}\end{array}$ \\
\hline II & $\begin{array}{l}\text { In front of Iron and } \\
\text { Steel factory }\end{array}$ & $3.66 \mathrm{Km}$ & $\begin{array}{l}\text { N: } 30^{\circ} 6^{\prime} 8,18 " \\
E: 31^{\circ} 16^{\prime} 9,62^{\prime \prime}\end{array}$ \\
\hline III & $\begin{array}{l}\text { In front of Starch and } \\
\text { Glucose factory }\end{array}$ & $5.71 \mathrm{Km}$ & $\begin{array}{l}\text { N: } 30^{\circ} 8^{\prime} 071^{\prime \prime} \\
E: 31^{\circ} 17^{\prime} 416^{\prime \prime}\end{array}$ \\
\hline IV & $\begin{array}{l}\text { In front of Petroleum } \\
\text { company }\end{array}$ & $6.0 \mathrm{Km}$ & $\begin{array}{l}\text { N: } 30^{\circ} 8^{\prime} 601^{\prime \prime} \\
E: 31^{\circ} 17^{\prime} 628^{\prime \prime}\end{array}$ \\
\hline V & $\begin{array}{l}\text { In front of Abu Za'baal } \\
\text { company for Fertilizer } \\
\text { and Chemical Products. }\end{array}$ & $22.96 \mathrm{Km}$ & $\begin{array}{l}\mathrm{N}: 30^{\circ} 16^{\prime} 132^{\prime \prime} \\
\mathrm{E}: 31^{\circ} 22^{\prime} 107^{\prime \prime}\end{array}$ \\
\hline VI & Anshas & $26.61 \mathrm{Km}$ & $\begin{array}{l}\text { N: } 30^{\circ} 18^{\prime} 518^{\prime \prime} \\
\text { E: } 31^{\circ} 23 \text { ' } 320^{\prime \prime}\end{array}$ \\
\hline
\end{tabular}

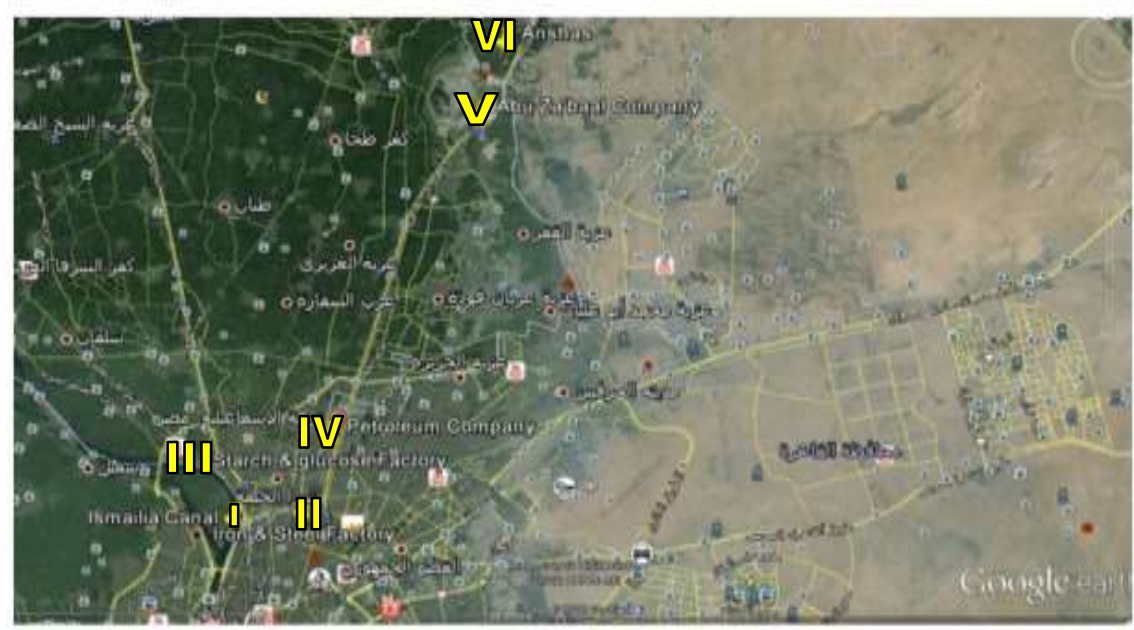

Fig (1) : Map showing the sampling sites along Ismailia Canal. 
Table (2): Variations of physico-chemical parameters of Ismailia Canal water during summer 2010.

\begin{tabular}{|c|c|c|c|c|c|c|c|}
\hline $\begin{array}{c}\text { Site } \\
\text { parameter }\end{array}$ & I & II & III & IV & $\mathbf{V}$ & VI & Mean \\
\hline $\mathbf{T}\left({ }^{\circ} \mathbf{C}\right)$ & 27.0 & 27.6 & 25.8 & 26.0 & 27.0 & 28.0 & 26.9 \\
\hline E.C $(\mu \mathrm{S} / \mathrm{cm})$ & 390 & 390 & 390 & 396 & 400 & 404 & 395.0 \\
\hline TDS (mg/L) & 195 & 195 & 195 & 198 & 200 & 202 & 197.5 \\
\hline pH & 7.8 & 7.8 & 7.6 & 7.6 & 7.6 & 7.6 & 7.66 \\
\hline Alkalinity $\left(\mathrm{mg} / \mathrm{L} \mathrm{CaCO}_{3}\right)$ & 184 & 188 & 172 & 184 & 170 & 188 & 181.0 \\
\hline $\mathrm{TH}\left(\mathrm{mg} / \mathrm{L} \mathrm{CaCO}_{3}\right)$ & 134 & 131 & 138 & 145 & 138 & 142 & 138 \\
\hline DO (mg/L) Winkler & 6.2 & 5.8 & 6.0 & 6.4 & 6.0 & 5.0 & 5.9 \\
\hline COD (mg/L) & 15.2 & 16.0 & 11.6 & 10.4 & 10.4 & 8.8 & 12.06 \\
\hline BOD (mg/L) & 1.0 & 1.5 & 1.8 & 1.8 & 2.0 & 1.5 & 1.6 \\
\hline $\mathrm{NH}_{3}(\mathrm{mg} / \mathrm{L})$ & 0.515 & 0.664 & 0.185 & 0.016 & 0.134 & 0.094 & $0.268 \pm$ \\
\hline $\mathrm{NO}_{2}^{-}(\mu \mathrm{g} / \mathrm{L})$ & 25.0 & 38.4 & 28.6 & 36.1 & 0.0 & 0.0 & 21.35 \\
\hline $\mathrm{NO}_{3}^{-}(\mathrm{mg} / \mathrm{L})$ & 0.0 & 0.0 & 0.1 & 0.7 & 0.4 & 0.3 & 0.25 \\
\hline $\mathrm{PO}_{4}{ }^{3-}(\mathrm{mg} / \mathrm{L})$ & 0.161 & 0.000 & 0.000 & 0.342 & 0.412 & 0.128 & 0.984 \\
\hline $\mathrm{Cl}^{-} \quad(\mathrm{mg} / \mathrm{L})$ & 16.4 & 15.3 & 12.2 & 12.5 & 15.3 & 15.6 & 14.6 \\
\hline $\mathrm{F}^{-} \quad(\mathrm{mg} / \mathrm{L})$ & 0.20 & 0.40 & 0.18 & 0.21 & 0.60 & 0.59 & 0.363 \\
\hline $\mathrm{Br}^{-}(\mathrm{mg} / \mathrm{L})$ & 0.60 & 0.20 & 0.08 & 0.7 & 0.12 & 0.16 & 0.205 \\
\hline $\mathrm{SO}_{4}{ }^{2-}(\mathrm{mg} / \mathrm{L})$ & 21.8 & 21.3 & 16.6 & 16.8 & 21.5 & 20.9 & 19.65 \\
\hline $\mathrm{Ca}^{2+}(\mathrm{mg} / \mathrm{L})$ & 28.1 & 24.0 & 31.0 & 31.5 & 31.3 & 31.6 & 29.6 \\
\hline $\mathrm{Mg}^{2+}(\mathrm{mg} / \mathrm{L})$ & 12.9 & 10.6 & 13.5 & 13.5 & 13.5 & 13.2 & 12.9 \\
\hline $\mathrm{Na}^{+} \quad(\mathrm{mg} / \mathrm{L})$ & 28.0 & 23.1 & 29.1 & 28.5 & 28.4 & 29.0 & 27.68 \\
\hline $\mathrm{K}^{+} \quad(\mathrm{mg} / \mathrm{L})$ & 4.35 & 3.89 & 5.21 & 5.15 & 5.90 & 5.59 & 5.02 \\
\hline Fe $\quad(\mathrm{mg} / \mathrm{L})$ Colorimetry & 0.25 & 013 & 0.07 & 0.09 & 0.07 & 0.41 & 0.17 \\
\hline Cd $(\mu \mathrm{g} / \mathrm{L})$ & 9.2 & 8.8 & 7.2 & 11.2 & 7.8 & 6.0 & 8.37 \\
\hline $\mathrm{Cu} \quad(\mu \mathrm{g} / \mathrm{L})$ & 38.5 & 0.0 & 8.0 & 2.9 & 2.2 & 0.0 & 8.6 \\
\hline $\mathrm{Zn}(\mu \mathrm{g} / \mathrm{L})$ & 40.0 & 0.0 & 0.0 & 0.0 & 0.0 & 0.0 & 6.67 \\
\hline $\mathrm{Pb}(\mu \mathrm{g} / \mathrm{L})$ & 0.0 & 0.0 & 0.0 & 0.0 & 0.0 & 0.0 & 0.0 \\
\hline
\end{tabular}


PHYSICO-CHEMICAL CHARACTERISTICS AND NATURAL .......

Table (3): Variations of physico-chemical parameters of Ismailia Canal water during autumn 2010.

\begin{tabular}{|c|c|c|c|c|c|c|c|}
\hline Site & $\mathbf{I}$ & II & III & IV & $\mathbf{V}$ & VI & Mean \\
\hline $\mathrm{T}^{\mathbf{O}} \mathrm{C}$ & 19.0 & 17.0 & 17.0 & 18.0 & 19.0 & 19.0 & 18.2 \\
\hline E.C $(\mu \mathrm{S} / \mathrm{cm})$ & 404 & 416 & 418 & 418 & 424 & 418 & 416.3 \\
\hline TDS (mg/L) & 202 & 208 & 209 & 209 & 212 & 209 & 208 \\
\hline pH & 7.8 & 7.8 & 8.0 & 7.8 & 7.6 & 7.6 & 7.76 \\
\hline Alkalinity (mg/L $\left.\mathrm{CaCO}_{3}\right)$ & 180 & 186 & 180 & 176 & 174 & 180 & 179.3 \\
\hline $\left.\mathrm{TH}(\mathrm{mg} / \mathrm{L} \mathrm{CaCO})_{3}\right)$ & 140 & 138 & 138 & 132 & 136 & 132 & 136 \\
\hline$\overline{\text { DO(mg/L) Winkler }}$ & 5.0 & 9.0 & 6.8 & 8.0 & 8.2 & 9.6 & 7.7 \\
\hline COD (mg/L) & 18.8 & 25.5 & 30.0 & 18.0 & 22.0 & 18.8 & 21.12 \\
\hline BOD $(\mathrm{mg} / \mathrm{L})$ & 1.5 & 1.4 & 2.6 & 2.4 & 2.0 & 3.3 & 2.2 \\
\hline $\mathrm{NH}_{3}(\mathrm{mg} / \mathrm{L})$ & 0.032 & 0.183 & 0.011 & 0.000 & 0.066 & 0.160 & 0.075 \\
\hline $\mathrm{NO}_{2}^{-}(\mathrm{mg} / \mathrm{L})$ & 44.7 & 50.6 & 46.0 & 38.4 & 38.4 & 46.6 & 44.11 \\
\hline $\mathrm{NO}_{3}^{-}(\mathrm{mg} / \mathrm{L})$ & 0.8 & 0.4 & 0.1 & 2.5 & 0.6 & 1.3 & 0.95 \\
\hline $\mathrm{PO}_{4}{ }^{3-}(\mathrm{mg} / \mathrm{L})$ & 0.206 & 0.000 & 0.146 & 0.000 & 0.557 & 0.523 & 0.107 \\
\hline $\mathrm{Cl}^{-} \quad(\mathrm{mg} / \mathrm{L})$ & 21.7 & 20.0 & 18.2 & 21.0 & 19.8 & 21.3 & 20.3 \\
\hline $\begin{array}{ll}\mathbf{F}^{-} & (\mathrm{mg} / \mathrm{L})\end{array}$ & 0.20 & 0.20 & 0.35 & 0.30 & 0.88 & 1.06 & 0.498 \\
\hline $\mathrm{Br}^{-}(\mathrm{mg} / \mathrm{L})$ & 0.00 & 0.00 & 0.00 & 0.30 & 0.00 & 0.00 & 0.050 \\
\hline $\mathrm{SO}_{4}{ }^{2-}(\mathrm{mg} / \mathrm{L})$ & 28.3 & 26.3 & 24.1 & 25.1 & 26.3 & 28.3 & 26.40 \\
\hline $\mathrm{Ca}^{2+}(\mathrm{mg} / \mathrm{L})$ & 27.9 & 23.8 & 30.2 & 31.5 & 31.4 & 31.6 & 29.4 \\
\hline $\mathrm{Mg}^{2+}(\mathrm{mg} / \mathrm{L})$ & 13.0 & 11.2 & 13.7 & 13.5 & 13.5 & 13.4 & 13.1 \\
\hline $\mathrm{Na}^{+} \quad(\mathrm{mg} / \mathrm{L})$ & 27.6 & 22.8 & 28.5 & 28.4 & 27.8 & 26.8 & 26.99 \\
\hline $\mathrm{K}^{+} \quad(\mathrm{mg} / \mathrm{L})$ & 4.40 & 3.90 & 4.20 & 5.10 & 5.20 & 5.40 & 4.70 \\
\hline $\begin{array}{ll}\mathrm{Fe} & (\mathrm{mg} / \mathrm{L}) \text { Colorimetry }\end{array}$ & 0.22 & 0.16 & 0.17 & 0.14 & 0.06 & 0.48 & 0.205 \\
\hline $\operatorname{Cd}(\mu \mathrm{g} / \mathrm{L})$ & 4.0 & 12.4 & 5.5 & 10.3 & 3.8 & 4.9 & 6.81 \\
\hline $\mathrm{Cu}(\mu \mathrm{g} / \mathrm{L})$ & 8.8 & 0.0 & 0.0 & 0.0 & 0.0 & 0.0 & 1.4 \\
\hline $\mathrm{Zn}(\mu \mathrm{g} / \mathrm{L})$ & 26.7 & 0.0 & 24.0 & 0.0 & 0.0 & 0.0 & 8.45 \\
\hline $\mathrm{Pb}(\mu \mathrm{g} / \mathrm{L})$ & 0.0 & 0.0 & 0.0 & 0.0 & 41.8 & 0.0 & 6.97 \\
\hline
\end{tabular}


Table (4): Variations of physico-chemical parameters of Ismailia Canal water during winter 2011.

\begin{tabular}{|c|c|c|c|c|c|c|c|}
\hline Site & $\mathbf{I}$ & II & III & IV & $\mathbf{V}$ & VI & Mean \\
\hline $\mathrm{T}^{\mathrm{O}} \mathrm{C}$ & 15.5 & 16.0 & 16.0 & 15.0 & 17.0 & 15.0 & 15.2 \\
\hline E.C $(\mu \mathrm{S} / \mathrm{cm})$ & 460 & 480 & 474 & 470 & 474 & 456 & 469 \\
\hline TDS(mg/L) & 230 & 240 & 237 & 235 & 237 & 228 & 234.5 \\
\hline pH & 7.6 & 7.4 & 8.0 & 8.0 & 7.4 & 7.0 & 7.56 \\
\hline Alkalinity (mg/L CaCO$\left.)_{3}\right)$ & 182 & 180 & 190 & 170 & 176 & 180 & 179.7 \\
\hline $\begin{array}{l}\text { TH } \\
\left(\mathrm{mg} / \mathrm{L} \mathrm{CaCO}_{3}\right)\end{array}$ & 144 & 152 & 152 & 142 & 148 & 140 & 146.5 \\
\hline DO(mg/L) Winkler & 8.0 & 10.6 & 9.0 & 9.0 & 10.2 & 10.4 & 9.53 \\
\hline COD $(m g / L)$ & 22.8 & 16.0 & 16.0 & 25.2 & 18.8 & 20.0 & 19.8 \\
\hline BOD $(\mathrm{mg} / \mathrm{L})$ & 3.4 & 3.6 & 3.3 & 3.7 & 3.0 & 2.4 & 3.23 \\
\hline $\mathrm{NH}_{3}(\mathrm{mg} / \mathrm{L})$ & 0.002 & 0.270 & 0.000 & 0.000 & 0.000 & 0.006 & 0.046 \\
\hline $\mathrm{NO}_{2}^{-}(\mathrm{mg} / \mathrm{L})$ & 46.0 & 58.1 & 37.4 & 42.0 & 47.0 & 46.0 & 46.08 \\
\hline $\mathrm{NO}_{3}{ }^{-}(\mathrm{mg} / \mathrm{L})$ & 0.5 & 1.0 & 0.8 & 1.4 & 0.5 & 1.0 & 0.87 \\
\hline $\mathrm{PO}_{4}{ }^{3-}(\mathrm{mg} / \mathrm{L})$ & 0.297 & 0.000 & 0.267 & 0.000 & 0.821 & 0.236 & 0.186 \\
\hline $\mathrm{Cl}^{-} \quad(\mathrm{mg} / \mathrm{L})$ & 40.0 & 40.2 & 40.4 & 40.0 & 41.0 & 40.3 & 40.3 \\
\hline $\begin{array}{ll}F^{-} & (\mathrm{mg} / \mathrm{L})\end{array}$ & 0.28 & 0.28 & 0.26 & 0.30 & 0.40 & 1.0 & 0.420 \\
\hline $\mathrm{Br}^{-}(\mathrm{mg} / \mathrm{L})$ & 0.29 & 0.35 & 0.27 & 0.26 & 0.26 & 0.21 & 0.273 \\
\hline $\mathrm{SO}_{4}{ }^{2-}(\mathrm{mg} / \mathrm{L})$ & 39.7 & 31.1 & 31.2 & 28.8 & 30.6 & 34.0 & 32.57 \\
\hline $\mathrm{Ca}^{2+}(\mathrm{mg} / \mathrm{L})$ & 27.7 & 30.7 & 31.2 & 28.6 & 30.4 & 25.9 & 29.1 \\
\hline $\mathrm{Mg}^{2+}(\mathrm{mg} / \mathrm{L})$ & 15.6 & 16.2 & 15.3 & 13.8 & 14.4 & 11.9 & 14.5 \\
\hline $\mathrm{Na}^{+} \quad(\mathrm{mg} / \mathrm{L})$ & 32.9 & 34.4 & 33.0 & 30.7 & 33.1 & 28.6 & 32.10 \\
\hline $\mathrm{K}^{+} \quad(\mathrm{mg} / \mathrm{L})$ & 4.99 & 4.98 & 5.00 & 4.60 & 4.97 & 4.20 & 4.79 \\
\hline $\begin{array}{ll}\mathrm{Fe} & (\mathrm{mg} / \mathrm{L}) \text { Colorimetry }\end{array}$ & 0.01 & 0.07 & 0.00 & 0.05 & 0.06 & 0.31 & 0.083 \\
\hline $\mathrm{Cd}(\mu \mathrm{g} / \mathrm{L})$ & 7.8 & 5.0 & 4.9 & 6.4 & 5.7 & 8.8 & 6.43 \\
\hline $\mathrm{Cu}(\mu \mathrm{g} / \mathrm{L})$ & 8.8 & 0.0 & 0.0 & 0.7 & 7.4 & 0.0 & 2.81 \\
\hline $\operatorname{Zn}(\mu \mathrm{g} / \mathrm{L})$ & 21.9 & 0.0 & 0.0 & 0.0 & 29.0 & 11.1 & 10.33 \\
\hline $\mathrm{Pb}(\mu \mathrm{g} / \mathrm{L})$ & 133.0 & 74.0 & 48.0 & 0.0 & 99.0 & 259.0 & 102.17 \\
\hline
\end{tabular}


PHYSICO-CHEMICAL CHARACTERISTICS AND NATURAL

Table (5): Variations of physico-chemical parameters of Ismailia Canal water during spring 2011.

\begin{tabular}{|c|c|c|c|c|c|c|c|}
\hline $\mathrm{P}_{\text {parameter }}^{\text {site }}$ & $\mathbf{I}$ & II & III & IV & $\mathbf{V}$ & VI & Mean \\
\hline $\mathrm{T}^{\mathrm{O}} \mathrm{C}$ & 22.0 & 20.0 & 20.5 & 21.0 & 21.0 & 22.0 & 21.1 \\
\hline E.C $(\mu \mathrm{S} / \mathrm{cm})$ & 360 & 372 & 270 & 365 & 367 & 390 & 354 \\
\hline TDS(mg/L) & 180 & 186 & 135 & 182.5 & 183.5 & 195 & 177 \\
\hline pH & 8.2 & 8.2 & 8.0 & 8.0 & 7.8 & 8.0 & 8.03 \\
\hline Alkalinity(mg/L CaCO 3$)$ & 170 & 174 & 176 & 172 & 180 & 174 & 174.3 \\
\hline $\mathrm{TH}\left(\mathrm{mg} / \mathrm{L} \mathrm{CaCO}_{3}\right)$ & 128 & 120 & 126 & 124 & 126 & 128 & 125.3 \\
\hline DO(mg/L) Winkler & 8.2 & 6.4 & 8.0 & 7.0 & 7.4 & 6.9 & 7.31 \\
\hline COD $(\mathrm{mg} / \mathrm{L})$ & 19.2 & 14.4 & 15.6 & 22.0 & 18.0 & 16.0 & 17.53 \\
\hline BOD $(\mathrm{mg} / \mathrm{L})$ & 1.0 & 2.0 & 0.0 & 2.0 & 3.5 & 2.0 & 1.75 \\
\hline $\mathrm{NH}_{3}(\mathrm{mg} / \mathrm{L})$ & 0.042 & 0.229 & 0.183 & 0.244 & 0.183 & 0.009 & 0.148 \\
\hline $\mathrm{NO}_{2}^{-}(\mathrm{mg} / \mathrm{L})$ & 0.0 & 46.0 & 34.8 & 0.0 & 22.7 & 27.6 & 21.85 \\
\hline $\mathrm{NO}_{3}^{-}(\mathrm{mg} / \mathrm{L})$ & 0.0 & 0.1 & 0.1 & 0.4 & 0.2 & 0.2 & 0.17 \\
\hline $\mathrm{PO}_{4}{ }^{3-}(\mathrm{mg} / \mathrm{L})$ & 0.252 & 0.000 & 0.237 & 0.206 & 0.495 & 0.155 & 0.120 \\
\hline $\mathrm{Cl}^{-} \quad(\mathrm{mg} / \mathrm{L})$ & 13.6 & 13.7 & 13.7 & 13.8 & 14.0 & 13.3 & 13.0 \\
\hline $\mathrm{F}^{-} \quad(\mathrm{mg} / \mathrm{L})$ & 0.34 & 0.28 & 0.22 & 0.26 & 0.73 & 0.39 & 0.37 \\
\hline $\mathrm{Br}^{-}(\mathrm{mg} / \mathrm{L})$ & 0.45 & 0.10 & 0.15 & 0.21 & 0.19 & 0.07 & 0.195 \\
\hline $\mathrm{SO}_{4}{ }^{2-}(\mathrm{mg} / \mathrm{L})$ & 20.1 & 20.4 & 19.3 & 20.8 & 21.3 & 21.4 & 20.55 \\
\hline $\mathrm{Ca}^{2+}(\mathrm{mg} / \mathrm{L})$ & 28.0 & 27.0 & 30.73 & 31.4 & 30.8 & 31.2 & 29.9 \\
\hline $\mathrm{Mg}^{2+} \quad(\mathrm{mg} / \mathrm{L})$ & 12.7 & 12.5 & 13.4 & 13.5 & 13.4 & 13.2 & 11.4 \\
\hline $\mathrm{Na}^{+} \quad(\mathrm{mg} / \mathrm{L})$ & 27.8 & 25.9 & 27.1 & 27.8 & 27.9 & 28.0 & 27.43 \\
\hline $\mathrm{K}^{+} \quad(\mathrm{mg} / \mathrm{L})$ & 4.51 & 4.13 & 3.90 & 5.18 & 5.20 & 5.50 & 4.73 \\
\hline $\begin{array}{ll}\mathrm{Fe} & (\mathrm{mg} / \mathrm{L}) \text { Colorimetry }\end{array}$ & 0.10 & 0.10 & 0.14 & 0.13 & 0.23 & 0.13 & 0.138 \\
\hline $\operatorname{Cd}(\mu \mathrm{g} / \mathrm{L})$ & 3.0 & 6.0 & 1.0 & 4.5 & 5.0 & 4.0 & 3.91 \\
\hline $\mathrm{Cu}(\mu \mathrm{g} / \mathrm{L})$ & 0.0 & 0.0 & 0.0 & 0.0 & 0.0 & 0.0 & 0.0 \\
\hline $\operatorname{Zn}(\mu \mathrm{g} / \mathrm{L})$ & 0.0 & 0.0 & 0.0 & 0.0 & 0.0 & 0.0 & 0.0 \\
\hline $\mathrm{Pb}(\mu \mathrm{g} / \mathrm{L})$ & 21.0 & 0.0 & 0.0 & 12.9 & 0.0 & 0.0 & 5.56 \\
\hline
\end{tabular}


Table (6) : The activity level concentration of ${ }^{40} \mathrm{~K},{ }^{238} \mathrm{U}$ and ${ }^{232} \mathrm{Th}$ in the investigated sites of Ismailia Canal water during 2010/2011 using $\gamma$-ray spectrometry.

\begin{tabular}{|c|c|c|c|}
\hline site & \multicolumn{1}{|c|}{${ }^{{ }^{40} \mathrm{~K} \pm \mathrm{E}}$} & ${ }^{238} \mathbf{U} \pm \mathrm{E}$ & $\begin{array}{l}{ }^{232} \mathrm{Th} \pm \mathrm{E} \\
\mathbf{B q} / \mathbf{L}\end{array}$ \\
\hline \hline I & $<\mathrm{DL}$ & $<\mathrm{DL}$ & $<\mathrm{DL}$ \\
\hline \hline II & $47.15 \pm 4.93$ & $3.64 \pm 1.4$ & $3.29 \pm 1.34$ \\
\hline \hline III & $98.14 \pm 3.6$ & $6.52 \pm 1.74$ & $2.66 \pm 0.82$ \\
\hline \hline V & $78.65 \pm 6.76$ & $6.97 \pm 1.02$ & $2.05 \pm 1.08$ \\
\hline \hline VI & $103.84 \pm 7.01$ & $3.8 \pm 1.34$ & $2.65 \pm 1.55$ \\
\hline
\end{tabular}

DL: detection limits for ${ }^{40} \mathrm{~K},{ }^{238} \mathrm{U}$ and ${ }^{232} \mathrm{Th}$ are 3.0, 0.6 and $0.7 \mathrm{~Bq} / \mathrm{L}$, respectively. $\mathbf{E H C W}^{(\mathbf{1 0})}$ (Egyptian Higher Committee of Water), Egyptian standards for drinking and domestic water.

Egyptian Law (48/1982) ${ }^{(\mathbf{1 1})}$, for the protection of the River Nile and water ways from pollution.

WHO $^{(\mathbf{1 2})}$ (World Health Organization); “Guidelines for drinking water quality.

EPA $^{(\mathbf{1 3})}$ (Environmental Protection Agency): National Drinking Water Regulations.

$\mathbf{E U}^{(\mathbf{1 4})}$ Council of the European Union) ; concern with the quality of water intended for human consumption.

a- no health based guideline.

b- Primary Maximum Contaminant Level - regulation is health-based.

c- Secondary Maximum Contaminant Level - regulation is based on aesthetic considerations .

Table (7): The results of water quality parameter of water of Ismailia Canal and the permissible limits.

\begin{tabular}{|c|c|c|c|c|c|c|}
\hline $\begin{array}{l}\text { Water quality } \\
\text { parameters }\end{array}$ & $\begin{array}{l}\text { Present } \\
\text { study }\end{array}$ & $\mathrm{EHCW}^{(18}$ & $\begin{array}{l}\text { Law } \\
{ }^{(19)} 48 / 1982\end{array}$ & $\begin{array}{l}\mathrm{WHO}^{(2} \\
0)\end{array}$ & $\mathrm{EPA}^{(21)}$ & $\mathrm{EU}^{(22)}$ \\
\hline E.C (mg/L) & $270-480$ & - & - & - & - & 2500 \\
\hline TDS (mg/L) & $135-240$ & - & - & $\mathrm{a}$ & $500^{(\mathrm{c})}$ & - \\
\hline $\mathrm{pH}$ & $7-8.2$ & - & - & $\mathrm{a}$ & $6.5-8.5^{(\mathrm{c})}$ & $6.5-9.5$ \\
\hline $\begin{array}{l}\text { Alkalinity }\left(\mathrm{CaCO}_{3}\right. \\
\mathrm{mg} / \mathrm{L})\end{array}$ & $170-190$ & - & - & - & - & - \\
\hline $\mathrm{DO}(\mathrm{mg} / \mathrm{L})$ & $5-10.6$ & - & $>5$ & - & - & - \\
\hline $\mathrm{COD}(\mathrm{mg} / \mathrm{L})$ & $8.8-30$ & - & $<15$ & - & - & - \\
\hline $\mathrm{BOD}(\mathrm{mg} / \mathrm{L})$ & $1-3.7$ & - & $<6$ & - & - & - \\
\hline Total hardness & $170-188$ & - & - & - & - & - \\
\hline
\end{tabular}


PHYSICO-CHEMICAL CHARACTERISTICS AND NATURAL

\begin{tabular}{|c|c|c|c|c|c|c|}
\hline$\left(\mathrm{CaCO}_{3} \mathrm{mg} / \mathrm{L}\right)$ & & & & & & \\
\hline $\mathrm{NH}_{3}(\mathrm{mg} / \mathrm{L})$ & $0-0.664$ & - & 0.5 & $\mathrm{a}$ & - & 0.5 \\
\hline $\mathrm{No}_{2}{ }^{-}(\mathrm{mg} / \mathrm{L})$ & $0-0.581$ & - & - & 3 & $1^{(\mathrm{b})}$ & 0.5 \\
\hline $\mathrm{No}_{3}{ }^{-}(\mathrm{mg} / \mathrm{L})$ & $0.0-2.5$ & - & 45 & 50 & $10^{(\mathrm{b})}$ & 50 \\
\hline $\mathrm{Po}_{4}{ }^{-3}(\mathrm{mg} / \mathrm{L})$ & $0.0-0.821$ & - & - & - & - & - \\
\hline $\mathrm{Cl}^{-}(\mathrm{mg} / \mathrm{L})$ & $12.2-40.4$ & - & - & $\mathrm{a}$ & $250^{(\mathrm{c})}$ & 250 \\
\hline $\mathrm{F}^{-}(\mathrm{mg} / \mathrm{L})$ & $0.2-1.06$ & 0.08 & - & 1.5 & $2^{(\mathrm{c})}-4^{(\mathrm{b})}$ & 1.5 \\
\hline $\mathrm{Br}^{-}(\mathrm{mg} / \mathrm{L})$ & $0-0.45$ & - & - & $\mathrm{a}$ & - & - \\
\hline $\mathrm{SO}_{4}{ }^{-2}(\mathrm{mg} / \mathrm{L})$ & $16.6-39.7$ & 400 & - & $\mathrm{a}$ & $250^{(\mathrm{c})}$ & 250 \\
\hline $\mathrm{Ca}^{+2}(\mathrm{mg} / \mathrm{L})$ & $23.8-35.3$ & 200 & - & $\mathrm{a}$ & - & - \\
\hline $\mathrm{Mg}^{+2}(\mathrm{mg} / \mathrm{L})$ & $10.2-16.1$ & 150 & - & $\mathrm{a}$ & - & - \\
\hline $\mathrm{Na}^{+}(\mathrm{mg} / \mathrm{L})$ & $23.1-34.4$ & 200 & - & $\mathrm{a}$ & - & 200 \\
\hline $\mathrm{K}^{+}(\mathrm{mg} / \mathrm{L})$ & $3.89-5.90$ & - & - & $\mathrm{a}$ & - & - \\
\hline $\mathrm{Fe}(\mathrm{mg} / \mathrm{L})$ & $0.0-0.48$ & - & - & $\mathrm{a}$ & $0.3^{(\mathrm{c})}$ & 0.2 \\
\hline $\mathrm{Cd}^{2+}(\mu \mathrm{g} / \mathrm{L})$ & $1-12.4$ & 5 & - & 3 & $5^{(\mathrm{b})}$ & 5 \\
\hline $\mathrm{Cu}^{2+}(\mu \mathrm{g} / \mathrm{L})$ & $0.0-38.5$ & 1000 & - & 2000 & $1300^{(\mathrm{b})}$ & 2000 \\
\hline $\mathrm{Zn}^{2+} \quad(\mu \mathrm{g} / \mathrm{L})$ & $0.0-40.0$ & 5000 & - & $\mathrm{a}$ & $5000^{(\mathrm{c})}$ & - \\
\hline $\mathrm{Pb}^{2+} \quad(\mu \mathrm{g} / \mathrm{L})$ & $0.0-259$ & 50 & - & 10 & $15^{(\mathrm{b})}$ & 10 \\
\hline $\mathrm{Al}^{3+} \quad(\mathrm{mg} / \mathrm{L})$ & $0.0-1.708$ & 0.2 & - & 0.2 & 0.2 & 0.2 \\
\hline${ }^{40} \mathrm{~K}(\mathrm{~Bq} / \mathrm{L})$ & $\begin{array}{l}0.0- \\
103.84\end{array}$ & - & - & - & - & - \\
\hline${ }^{238} \mathrm{U}(\mathrm{Bq} / \mathrm{L})$ & $0.0-6.97$ & - & - & 10 & $\begin{array}{c}0.372 \\
(30 u g / L)\end{array}$ & - \\
\hline${ }^{232} \mathrm{Th}(\mathrm{Bq} / \mathrm{L})$ & $0.0-3.65$ & - & - & 1 & - & - \\
\hline
\end{tabular}

\section{References}

1. Nair, I.V.; Singh, K.; Arumugam, M.; Gangadhar, K. and Clarson, D. 2010. Trace metal quality of Meenachil River at Kottayam, Kerala (India) by principal component analysis. World Appl. Sci. J. 9(10): 1100-1107.

2. Geriesh, M.H.; Balke, K. and El-Rayes, A. 2008. Problems of drinking water treatment along Ismailia Canal Province, Egypt. J., Zhejiang Univ. Sci. B., 9 (3): 232-242.

3. Abdo, M. H. ; Sabae, S. Z.; Haroon, B. M.; Refaat, B.M.and Mohammed, A.S. 2010. Physico-chemical characteristics, microbial assessment and antibiotic susceptibility of pathogenic bacteria of Ismailia Canal water, Egypt. J. Amer. Sci., 6(5) :234-250.

4. Abd El-Hady, H.H. and Hussian ,A.M . 2012. Regional and seasonal variation of phytoplankton assemblages and its biochemical analysis in Ismailia Canal, River Nile, Egypt. J. Appl. Sci. Res., 8(7): 3433-3447. 
5. Stahl, R. and Ramadan, A.B. 2008. Environmental studies on water quality of the Ismailia Canal / Egypt. WissenschaftlicheBerichte FZKA, 7427: 1-47.

6. Mollah, A.S.; Rahman, M.M. and Husain, S.R. 1986. Distribution of gammaemitting radionuclides in soils at the Atomic Energy Research Establichment, Savar, Bangladesh. J. Health Phys., 50 (6): 835-838.

7. American Public Health Association (APHA), American Water Works Association (AWWA) and Water Environment Federation (WEF). 2005. "Standard methods for the examination of water and wastewater" $21^{\text {st }}$ Ed. American Public Health Association, Washington, DC. .

8. Dionex Corporation. 1998. Determination of transition metals at ppt levels in high-purity water and SC2 (D-clean) baths. Application Note 131, LPN 1058-01, Sunnyvale, CA.

9. Abdo, M. H. and El-Nasharty, S. M . 2010. Physico-chemical evaluations and trace metals distribution in water-surficial sediment of Ismailia Canal, Egypt. J. Nat . Sci., 8(5): 198206.

10. EHCW (Egyptian Higher Committee of Water). 1995. Egyptian standards for drinking and domestic water according to the Act 27/1978 in regulating of the public water supplies. Egyptian Governmental Press.

11. Egyptian Law (48/1982). The Implementer Regulations for law 48/1982 regarding the protection of the River Nile and water ways from pollution. Map. Periodical Bull, pp 12-35.

12. WHO (World Health Organization). 2008. "Guidelines for drinking water quality" $3^{\text {rd }}$ Ed. WHO, Geneva.

13. EPA (Environmental Protection Agency). 2009. National Drinking Water Regulations. http://water.epa.gov/drink/contaminants/index.cfm

14. Council of the European Union. 1998. Council directive 98/83/EC on the quality of water intended for human consumption.Official Journal of the European Communities, No L330/54 pp 32-52.

15. Abdo, M. H. 1998. Some environmental studies on the River Nile and Ismailia Canal in front of the industrial area of Shoubra El-Kheima. M. Sc. Thesis, Faculty. Science, Ain Shams University, Egypt.

16. Abdel-Satar, A. M . 2005. Water quality assessment of River Nile from Idfo to Cairo, Egypt. J. Aqua. Res., 31 (2): 200-223.

17. Sabae, S.Z. 2004. Monitoring of microbial pollution in the River Nile and the impact of someHuman activities on its waters. Proc. 3rd Int. Conf. Biol. Sci. Fac. Sci. Tanta Univ., 28-29 April, 3: 200-214.

18. Abdel Malik, W.E.Y.; Ibrahim, A.S.; Youssef, S.K.; Aziz, M. and El-Shinawy, R.M.K. 2010. Radiological review studies on Ismailia Canal ecology.Tenth Radiation Physics \& Protection Conference, Cairo, 27-30 November.

19. Murugesan,A. G. and Rajakumari, C. 2005. "Environmental science and biotechnology",M.J.P. Publication,Chennai.

20. Jayakumar, P.; Jothivel, N.; Thimmappa, A. And Paul, V.I. 2009. Physicochemical characterization of a lentic water body from Tamil Nadu with special reference to its pollution status .The ECOSCAN., 3(1\&2):59-64 
21. Abd-El-Razak, A. B.; Asiedu, R. E. M. and de-Graft- Johnson, K. A. A. 2009. Assessment of the water quality of the Oti River in Ghana.West Afric.J. Appl. Ecology., 15: 45-60.

22. EPA (Environmental Protection Agency).2001."Parameters of water quality-interpretation and standards" $3^{\text {rd }} E d$. EPA, Ireland.

23. Igbinosa, E. O. and Okoh, A. I. 2009. Impact of discharge wastewater effluents on the physico-chemical qualities of a receiving watershed in a typical rural community.Community Int. J. Environ. Sci. Tech., 6 (2): 175-182.

24. CCME (Canadian Council of Ministers of the Environment). 2010 Canadian water quality guidelines for the protection of aquatic life: ammonia.ceqgrcqe.ccme.ca/download/en/141/

25. Wetzel, R. G.1983.“Limnology”.2nd Ed. Saunder College Publishing, pp 767.

26. Abdo, M . H. 2005. Physico-chemical characteristics of Abu Za'baal ponds, Egypt. Egypt. J. Aquatic. Res., 31(2): 1-15.

27. Sabae, S.Z. and Abdel-Satar, A.M. 2001.Chemical and bacteriological studies on ElSalam Canal, Egypt. J. Egypt. Acad. Soc. Environ. Develop., 2(1): 173-197.

28. Haamer, K. 2006. Hydrochemistry and sources of fluoride in Silurian-. Ordovician aquifer system. M. Sc. Thesis, Institute. Geology, University. Tartu., Estonia.

29. Arlow, A. 2003. Crystallization aspects of the wet- process phosphoric acid industry.M. Sc. Thesis, Faculty. Engineering.,University.Pretoria., Pretoria,2003 .http://upetd.up.ac.za/thesis/available/etd04152004095501/unrestricted/00Front.pdf

30. Toufeek, M.E.F. 2011.Distribution of cadmium and lead in Aswan Reservoir and River Nile water at Aswan.World Appl. Sci. J. 13(2):369-375.

31. Smith, K.P. 1992.An overview of naturally occurring radioactive materials (NORM) in the petroleum industry. Enviromental Assessment and Information Science Division.

32. Kalra, N.; Kumar, R.; Yadav, S.S. and Singh, R.T.2012. Water quality index assessment of ground water in Koilwar block of Bhojpur (Bihar) J. Chem. Pharm. Res. 4(3):1782-1786.

33. Canadian Council of Minister of the Environment (CCME) .2001. Canadian water quality guidelines for the protection of aquatic life:CCME Water Quality Index 1.0, Technical Report," Canadian Council of Ministers of the Environment Winnipeg MB, Canada. 
